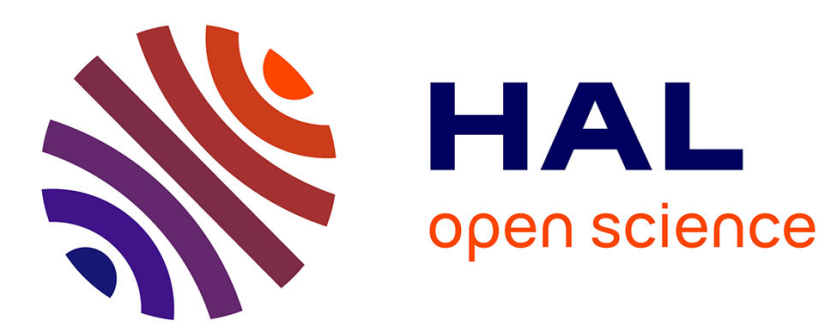

\title{
Language constrained stabilization of discrete-time switched linear systems: a Lyapunov-Metzler inequalities approach
}

\author{
Marc Jungers, Antoine Girard, Mirko Fiacchini
}

\section{To cite this version:}

Marc Jungers, Antoine Girard, Mirko Fiacchini. Language constrained stabilization of discrete-time switched linear systems: a Lyapunov-Metzler inequalities approach. CDC 2016 - 55th IEEE Conference on Decision and Control, Dec 2016, Las Vegas, NV, United States. 10.1109/cdc.2016.7799120 . hal-01386851

\section{HAL Id: hal-01386851 \\ https://hal.science/hal-01386851}

Submitted on 22 Jul 2020

HAL is a multi-disciplinary open access archive for the deposit and dissemination of scientific research documents, whether they are published or not. The documents may come from teaching and research institutions in France or abroad, or from public or private research centers.
L'archive ouverte pluridisciplinaire HAL, est destinée au dépôt et à la diffusion de documents scientifiques de niveau recherche, publiés ou non, émanant des établissements d'enseignement et de recherche français ou étrangers, des laboratoires publics ou privés. 


\title{
Language constrained stabilization of discrete-time switched linear systems: a Lyapunov-Metzler inequalities approach
}

\author{
Marc Jungers, Antoine Girard and Mirko Fiacchini
}

\begin{abstract}
This paper addresses the issue of stabilizability of an autonomous discrete-time switched system via a switching law that is constrained to belong to a language generated by an nondeterministic finite state automaton. Firstly the automaton is decomposed into strongly connected components to reduce the problem to the stabilizability of each non trivial strongly connected component. Secondly the approach considering Lyapunov-Metzler inequalities taking into account the language constraint for a strongly connected component is proposed. Links with the current literature are discussed and a detailed example is given to illustrate our contributions.
\end{abstract}

\section{INTRODUCTION}

Discrete-time switched systems are the association of a finite set of difference equations with a switching law that defines the active mode at each time [16]. They are a particular case of hybrid systems. Switched systems are particularly popular in control system theory due firstly to their ability to model real applications in broad fields and secondly to the richness and complexity of their behaviors. For instance, the stability analysis of switched systems with arbitrary switching laws is not intuitive and has motivated a great range of contributions, see [2], [11], [17] and references therein for more details.

The issue of designing a stabilizing switching law is also of interest and several results are provided in the literature [23]. Among them, one can cite the min-switching strategy associated with Lyapunov-Metzler inequalities [10]; the invariant set approach offering necessary and sufficient conditions for the stabilizability [8] or sufficient conditions based on LMIs [7]; the piecewise quadratic control-Lyapunov function approach (and its link with switched LQR problem) [26] and finally the feedback stabilization expressed via the joint spectral subradius [13].

Nevertheless switched systems should take into account constraints to be more in accordance with applications. That induces increased difficulties in their studies, which are the focus of recent contributions [3]. The constraints may be numerous and here we will consider only constraints concerning the admissible switching laws, like dwell-time

M. Jungers is with Université de Lorraine, CRAN, UMR 7039, 2 avenue de la forêt de Haye, Vandœuvre-lès-Nancy Cedex, 54516, France and CNRS, CRAN, UMR 7039, France. (marc. jungers@univ-lorraine.fr)

A. Girard is with Laboratoire des signaux et systèmes (L2S), CNRS, CentraleSupélec, Université Paris-Sud, Université ParisSaclay, 3, rue Joliot-Curie, 91192 Gif-sur-Yvette, cedex, France, (Antoine.Girardel2s.centralesupelec.fr)

M. Fiacchini is with Univ. Grenoble Alpes, GIPSA-lab, F-38000 Grenoble, France and CNRS, GIPSA-lab, F-38000 Grenoble, France. (mirko.fiacchiniegipsa-lab.fr)

This work was partially supported by project ANR COMPACS - "Computation Aware Control Systems", ANR-13-BS03-004. constraints (the duration between two consecutive switches should be greater than a given value), or modal constraints (some transitions from one mode to another are forbidden).

Stability of switched systems with constrained switching laws has been already investigated. Generally it consists in restricting the class of admissible switching laws [18]. Let us cite the case of (average) dwell-time constraints [12] and the modal constraints defined via a directed graph [25] treated via path depending Lyapunov functions [15] and recently via the constrained joint spectral radius [19]. Notice also the issue of determining an upper bound on dwell time allowing the stability [14].

Recent works deal with the stabilization of switched systems with constraints on the switching law. The paper [6] is focused on a modified min-switching strategy taking into account a fixed dwell-time, [9] provides conditions based on invariant set theory for language constrained switching laws. A generic formulation to cope with large classes of constraints is to specify a constrained language with a nondeterministic automaton [1] and to impose that the admissible switching laws belong to this language. Automaton decomposition techniques can thus be generically used to check whether an abstract property holds for a hybrid system or at least propagates in the automaton [21].

This paper considers the issue of stabilizability of a discrete-time switched system with switching laws belonging to the language generated by a nondeterministic automaton. The contribution of this paper is twofold. Firstly the automaton is decomposed into strongly connected components in order to simplify methodologically the stabilizability problem. Secondly a Lyapunov-Metzler inequalities approach taking into account the language is considered per strongly connected component to check the stabilizability of the switched system. When such Lyapunov-Metzler inequalities exist for at least one strongly connected component, a control policy is determined via a min-switching strategy.

The paper is organized as follows. Section II formalizes the problem of stabilizability. Section III gathers the definitions of useful tools that allow the generic methodology to investigate the stabilizability in Section IV. The main result concerning the Lyapunov-Metzler inequalities approach for a strongly connected component is provided in Section V, with a discussion establishing the link with the literature of stabilizability without language constraints. Detailed illustrations are provided in Section VI to emphasize the efficiency of our approach before concluding remarks in Section VII.

Notation: The notation is standard. Given $n \in \mathbb{N}$, define $\mathbb{N}_{n}=\{x \in \mathbb{N}: 1 \leq x \leq n\}$. Given $\alpha \in \mathbb{R}^{n}, \alpha_{i}$ denote 
its $i$-th element; given $\pi \in \mathbb{R}^{n \times m}, \pi_{i j}$ is the entry of $i$-th row and $j$-th column. $A^{\prime}$ denotes the transpose of matrix $A$. $I_{n}\left(0_{n \times p}\right)$ is the $n$-order identity matrix $(n \times p$-order null matrix). For a finite set $\mathcal{S},|\mathcal{S}|$ denotes its cardinality.

\section{PRoblem Formulation} form

We consider a discrete-time switched linear system of the

$$
x_{k+1}=A_{\sigma(k)} x_{k},
$$

where $x_{k} \in \mathbb{R}^{n}$ is the state at time $k \in \mathbb{N}$ and $\sigma: \mathbb{N} \rightarrow \mathbb{N}_{q}$ is the switching law and $\left\{A_{i}\right\}_{i \in \mathbb{N}_{q}}$, with $A_{i} \in \mathbb{R}^{n \times n}$ for all $i \in \mathbb{N}_{q}$. Given the initial state $x_{0}$ and a switching law $\sigma(\cdot)$, we denote with $x_{N}^{\sigma}\left(x_{0}\right)$ the state of the system (1) at time $N$ starting from $x_{0}$ by applying the switching law $\sigma(\cdot)$.

In this paper, we impose the constraint that the switching law $\sigma(\cdot)$ has to be an element of the language specified by a nondeterministic finite automaton [1].

Definition 1: A nondeterministic finite automaton is a tuple $\mathcal{A}=\left(\mathcal{S}, \Sigma, \delta, \mathcal{S}^{0}\right)$ where $\mathcal{S}$ is a finite set of automaton states; $\Sigma$ is a finite set of labels for the transitions; $\delta$ : $\mathcal{S} \times \Sigma \rightarrow 2^{\mathcal{S}}$ is a set-valued transition map, and $\mathcal{S}^{0} \subseteq \mathcal{S}$ is a subset of initial states.

Here the automaton is specified as follows. The labels are the modes of the system (1) and $\Sigma=\mathbb{N}_{q} \cdot \mathcal{S}$ is a set of $p=|\mathcal{S}|$ automaton states and we will denote $\mathcal{S}=\left\{e_{i}\right\}_{i \in \mathbb{N}_{p}}$ to avoid confusion with the elements of $\Sigma$.

Definition 2: A state $s \in \mathcal{S}$ is said to be reachable from state $\tilde{s} \in \mathcal{S}$ if $s=\tilde{s}$ or if there exists a finite sequence $s^{0}, s^{1}, \ldots, s^{N}$ such that $s^{0}=\tilde{s}, s^{N}=s$ and for all $k \in\{0, \ldots, N-1\}$, there exists $\sigma_{k} \in \Sigma$ such that $s_{k+1} \in \delta\left(s_{k}, \sigma_{k}\right)$. A state $s \in \mathcal{S}$ is said to be non-blocking if there exists $\sigma \in \Sigma$ such that $\delta(s, \sigma) \neq \emptyset$, otherwise it is said to be blocking.

A switching law $\sigma: \mathbb{N} \rightarrow \Sigma$ is said to belong to the language of $\mathcal{A}$ denoted $\sigma \in L(\mathcal{A})$ if there exists $s: \mathbb{N} \rightarrow \mathcal{S}$ such that $s(0) \in \mathcal{S}^{0}$ and for all $k \in \mathbb{N}, s_{k+1} \in \delta\left(s_{k}, \sigma(k)\right)$.

In this paper, we consider the following notion of control policy and stabilizability.

Definition 3: A control policy $\nu: \mathbb{R}^{n} \times \mathcal{S} \rightarrow \Sigma \times \mathcal{S}$ is such that, for any $(x, r) \in \mathbb{R}^{n} \times \mathcal{S}$,

$$
\begin{aligned}
\nu(x, r) & =(i(x, r), s(x, r)) \\
& \in \Sigma \times \delta(r, i(x, r)), \text { with } \delta(r, i(x, r)) \neq \emptyset .
\end{aligned}
$$

Roughly speaking, $i(x, r)$ is the mode to apply and $s(x, r)$ the following automaton state. This implicit definition could be split into two steps: firstly select $i(x, r)$ such that $\delta(r, i(x, r)) \neq \emptyset$. Secondly select $s(x, r) \in \delta(r, i(x, r))$.

Definition 4: The system (1) is globally exponentially stabilizable relatively to the language $L(\mathcal{A})$ via a control policy $\nu$, if there are $c \geq 0, \lambda \in[0,1)$ and $s \in \mathcal{S}^{0}$, such that for all $x \in \mathbb{R}^{n}$, every switching law $\sigma \in L(\mathcal{A})$ generated by the first component of the control policy $\nu$ starting from $(x, s)$ verifies $\left\|x_{k}^{\sigma}(x)\right\| \leq c \lambda^{k}\|x\|$.

The problem investigated here is formalized as follows.
Problem 1: Let us consider the system (1) and the automaton $\mathcal{A}$ defining the language constraints for the switching laws. Determine a control policy $\nu$ that generates switching laws $\sigma \in L(\mathcal{A})$ verifying the language constraints and that globally exponentially stabilizes the closed-loop system (1).

Roughly speaking solving Problem 1 consists in obtaining sufficient conditions on the set of initial states of the automaton $\mathcal{S}_{0}$ and a strategy to build under conditions a switching law that globally exponentially stabilizes the system (1). Our approach in this paper is based on a min-switching strategy associated with Lyapunov-Metzler inequalities taking into account the language constraints of the switching laws.

\section{PRELIMINARIES}

In this section, we first introduce several classes of matrices and tools related to the nondeterministic finite automaton $\mathcal{A}$ (see for example [4], [20]).

From the definition of a stochastic matrix, which is a square matrix with nonnegative entries and all row sums are equal to 1 , we extend the definition as follows.

Definition 5: A real-valued rectangular matrix is called a rectangular stochastic one, if all its components are nonnegative and the sum of each row is unitary. The set of rectangular stochastic matrices of dimension $n_{1} \times n_{2},\left(n_{1}, n_{2}\right) \in \mathbb{N}^{2}$, is denoted $\mathcal{M}_{n_{1} \times n_{2}} \subset \mathbb{R}^{n_{1} \times n_{2}}$.

Remark 1: For the min-switching strategy of switching system (without constraints) in discrete-time [10] a square Metzler matrix (with its off-diagonal components nonnegative) is crucial. It is only a stochastic matrix in discrete-time. In the whole paper, we will call the rectangular stochastic matrices also rectangular Metzler matrices with abuse of language for this reason.

With the automaton $\mathcal{A}$, it is possible to associate the finite directed graph or digraph $\mathcal{G}=(\mathcal{V}, \mathcal{E})$, for which the set of vertices is the finite set of states $\mathcal{V}=\mathcal{S}$ and the set of edges is related to the transitions map $\delta: \mathcal{E}=\left\{(s, r) \in \mathcal{S}^{2}, \exists \ell \in\right.$ $\Sigma, r \in \delta(s, \ell)\}$.

Let us give some definitions related to the digraph $\mathcal{G}$ and its strongly connected components.

Definition 6: Let $(s, r) \in \mathcal{V}^{2} . s$ and $r$ are strongly connected if $s=r$ or if there exist a directed path from $s$ to $r$ and a directed path from $s$ to $r$. This relation between nodes is reflexive, symmetric and transitive and it is then an equivalence relation on the nodes.

This equivalence relation allows to partition $\mathcal{V}$ of $\mathcal{G}$ into disjoint sets called Strongly Connected Components defined in Definition 7.

Definition 7: Strongly Connected Components (SCCs). Let $\mathcal{G}=(\mathcal{V}, \mathcal{E})$ be a finite digraph and $\mathcal{C} \subset \mathcal{V} . \mathcal{C}$ is strongly connected if for every pair of vertices $(s, r) \in \mathcal{C}^{2}, s$ and $r$ are strongly connected. A strongly connected component (SCC) of the digraph $\mathcal{G}$ is a maximally strongly connected set of vertices. This is an equivalence class for the relation of strongly connectivity. In other words, $\mathcal{C}$ is an SCC if $\mathcal{C}$ is strongly connected and if there does not exists a SCC distinct from $\mathcal{C}$ which includes $\mathcal{C}$. A SCC $\mathcal{C}$ is called trivial 
if $\mathcal{C}=\{s\}$ and $(s, s) \notin \mathcal{E}$. A SCC $\mathcal{C}$ is called terminal if there is no $\operatorname{SCC} \mathcal{D} \neq \mathcal{C}$ such that $(s, r) \in \mathcal{E}$ for some $s \in \mathcal{C}$ and $r \in \mathcal{D}$. Finite digraph $\mathcal{G}$ is cyclic if and only if $\mathcal{G}$ contains a nontrivial SCC.

Every vertex of the digraph $\mathcal{G}$ belongs to one and only one SCC: the SCCs are equal or disjoint. This is not necessarily the case for its edges. In the following, we will denote $\mathcal{C}_{i}^{\mathcal{G}}$ $\left(i \in \mathbb{N}_{d}\right)$ the $d$ SCCs of the digraph $\mathcal{G}$ and $p_{i}=\left|\mathcal{C}_{i}^{\mathcal{G}}\right|$. We define the condensation of $\mathcal{G}$ as follows.

Definition 8: The condensation of a digraph $\mathcal{G}$ is a digraph $\mathcal{G}^{\mathrm{SCC}}=\left(\mathcal{V}^{\mathrm{SCC}}, \mathcal{E}^{\mathrm{SCC}}\right)$ built as follows. Each $\mathrm{SCC} \mathcal{C}_{i}^{\mathcal{G}}$ is contracted into a single vertex, that is $\left|\mathcal{V}^{\mathrm{SCC}}\right|=d$. The set of condensated edges $\mathcal{E}^{\mathrm{SCC}}$ consists in the directed edges of $\mathcal{G}$ making the link between the SCCs. Formally if $\mathcal{G}$ admits $d \mathrm{SCCs}, \mathcal{V}^{\mathrm{SCC}}=\left\{e_{1}^{\mathrm{SCC}}, \cdots, e_{d}^{\mathrm{SCC}}\right\}$ and

$$
\begin{gathered}
\mathcal{E}^{\mathrm{SCC}}=\left\{\left(e_{i}^{\mathrm{SCC}}, e_{j}^{\mathrm{SCC}}\right) \in \mathcal{V}^{\mathrm{SCC}} \times \mathcal{V}^{\mathrm{SCC}}, \exists\left(i_{0}, j_{0}\right) \in \mathcal{S}^{2},\right. \\
\left.\left(e_{i_{0}}, e_{j_{0}}\right) \in \mathcal{C}_{i} \times \mathcal{C}_{j}, \quad \exists \ell \in \Sigma, e_{j_{0}} \in \delta\left(e_{i_{0}}, \ell\right)\right\} .
\end{gathered}
$$

The condensation $\mathcal{G}^{\mathrm{SCC}}$ is a Directed Acyclic Graph (DAG), because it does not contain any cycle. We can define a relation between the SCCs as $\mathcal{C}_{i} \succeq \mathcal{C}_{j}$ if there exists a path between one vertex in $\mathcal{C}_{i}$ and a vertex in $\mathcal{C}_{j}$. This is a partial relation order between the SCCs because it is reflexive $\left(\mathcal{C}_{i} \succeq \mathcal{C}_{i}\right)$, antisymmetric $\left(\mathcal{C}_{i} \succeq \mathcal{C}_{j}\right.$ and $\mathcal{C}_{j} \succeq \mathcal{C}_{i}$ imply $\left.\mathcal{C}_{i}=\mathcal{C}_{j}\right)$ and transitive $\left(\mathcal{C}_{i} \succeq \mathcal{C}_{j}\right.$ and $\mathcal{C}_{j} \succeq \mathcal{C}_{\ell}$ imply $\left.\mathcal{C}_{i} \succeq \mathcal{C}_{\ell}\right)$

Computing the condensation of a digraph is a standard task and several dedicated algorithms are available in the literature. Among them, one can cite the Kosaraju's algorithm [22] or the Tarjan's algorithm [24].

Let us define a rectangular Metzler matrix admissible to a SCC of the automaton $\mathcal{A}$.

Definition 9: Let $\mathcal{A}=\left(\mathcal{S}, \Sigma, \delta, \mathcal{S}_{0}\right)$, with $\Sigma=\mathbb{N}_{q}$ and $\mathcal{C}=\left\{c_{1}, \cdots, c_{h}\right\}$ one of its nontrivial SCC.

$\Pi \in \mathbb{R}^{h q \times h}$ is a said to be a rectangular Metzler matrix admissible with the $\operatorname{SCC} \mathcal{C}$, if $\Pi^{\prime} \in \mathcal{M}_{q \times h q}$ and

$$
\forall(i, j, \ell) \in \mathbb{N}_{h}^{2} \times \mathbb{N}_{q}, c_{i} \notin \delta\left(c_{j}, \ell\right) \cap \mathcal{C} \Rightarrow \pi_{i+(\ell-1) q, j}=0 .
$$

Remark 2: The relation (4) may be viewed as a layout on the rectangular Metzler matrix $\Pi$.

Example 1: Let us consider the automaton $\mathcal{A}$ defined by Figure 1 , where $\mathcal{S}=\left\{e_{1}, e_{2}\right\}(p=2), \Sigma=\{1,2\}(q=2)$, $\delta\left(e_{1}, 1\right)=\left\{e_{2}\right\}, \delta\left(e_{2}, 1\right)=\left\{e_{1}, e_{2}\right\}, \delta\left(e_{2}, 2\right)=\left\{e_{2}\right\}$ and $\mathcal{S}_{0}=\left\{e_{1}\right\}$.

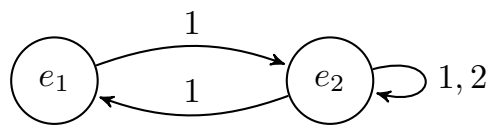

Fig. 1. Nondeterministic graph, Example 1.

This is a nondeterministic automaton because $\delta\left(e_{2}, 1\right)$ is not reduced to a singleton. $\mathcal{A}$ admits a single SCC $\mathcal{C}_{1}=\left\{e_{1}, e_{2}\right\}$. An admissible rectangular Metzler matrix $\Pi \in \mathbb{R}^{4 \times 2}$ associated with $\mathcal{C}_{1}$ is of the form

$$
\Pi=\left(\begin{array}{cccc}
0 & 1 & 0 & 0 \\
\alpha & \beta & 0 & 1-\alpha-\beta
\end{array}\right)^{\prime},
$$

with scalars $\alpha \geq 0, \beta \geq 0$ such that $\alpha+\beta \leq 1$.

\section{GENERIC APPROACH FOR LANGUAGE CONSTRAINED STABILIZATION}

First of all, we are interested in looking for switching laws of infinite length satisfying Proposition 2 . We denote the state of the constrained switched system as the concatenation of the state $x_{k} \in \mathbb{R}^{n}$ of the system (1) and the state of the automaton $s_{k} \in \mathcal{S}$. The trajectory is thus the function $k \mapsto$ $\left(x_{k}, s_{k}\right)$, with $k \in \mathbb{N}$. For a given trajectory $k \mapsto\left(x_{k}, s_{k}\right)$, the function $k \mapsto s_{k}$ is called its projection on the automaton state space and $k \mapsto x_{k}$ its projection on the system state space.

Proposition 2: Let $\mathcal{G}$ be the digraph associated with the automaton $\mathcal{A}$ and its condensation $\mathcal{G}^{\text {SCC }}$. Every trajectory of the constrained switched system in the sense of Definition 4 has a projection on the automaton state that ultimately enters and does not exit a non trivial SCC.

Proof: The proof is obtained thanks to the fact that the condensation is a DAG.

The first corollary of Proposition 2 is that without loss of generality, we can build an automaton $\tilde{\mathcal{A}}$ based on the automaton $\mathcal{A}$ such that $L(\mathcal{A})=L(\tilde{\mathcal{A}})$ and $\tilde{\mathcal{A}}$ has only non trivial terminal SCC. This starts from $\tilde{\mathcal{A}}=\mathcal{A}$ and erases iteratively all the trivial terminal SCC. This is equivalent to consider Assumption 1.

Assumption 1: For all $s \in \mathcal{S}, s$ is non-blocking.

Guided by Proposition 2, we introduce the implicit definition of control policy admissible with a SCC and the definition of exponentially stabilizing control Lyapunov function for switched systems, inspired by [26] and that are defined only on a restriction of the automaton state set $\mathcal{S}$. See also [9].

Definition 10: Let $\mathcal{C}$ be a nontrivial SCC induced by the automaton $\mathcal{A}$. A control policy over $\mathcal{C}$ is defined by $\nu^{\mathcal{C}}:$ $\mathbb{R}^{n} \times \mathcal{C} \rightarrow \Sigma \times \mathcal{C}$ such that, for any $(x, r) \in \mathbb{R}^{n} \times \mathcal{C}$,

$$
\begin{aligned}
\nu^{\mathcal{C}}(x, r) & =(i(x, r), s(x, r)) \in \Sigma \times(\delta(r, i(x, r)) \cap \mathcal{C}), \\
& \text { with } \delta(r, i(x, r)) \cap \mathcal{C} \neq \emptyset .
\end{aligned}
$$

(notice that since $\mathcal{C}$ is a nontrivial $\mathrm{SCC}$, there always exists a label $i(x, r)$ such that $\delta(r, i(x, r)) \cap \mathcal{C} \neq \emptyset$.)

Definition 11: Let us consider a nontrivial $\operatorname{SCC} \mathcal{C}$ of the directed digraph $\mathcal{G}$ induced by the automaton $\mathcal{A}$. A nonnegative continuous function $V: \mathbb{R}^{n} \times \mathcal{C} \rightarrow \mathbb{R}^{+}$is an exponentially stabilizing control Lyapunov function (ECLF) of the system (1) in $\mathcal{C}$ if for any $(x, r) \in \mathbb{R}^{n} \times \mathcal{C}$, we have

1) $\kappa_{1}\|x\|^{2} \leq V(x, r) \leq \kappa_{2}\|x\|^{2}$ for some finite positive constants $\kappa_{1}$ and $\kappa_{2}$;

2) $V\left(A_{i(x, r)} x, s(x, r)\right)-V(x, r) \leq-\kappa_{3}\|x\|^{2}$, for a constant $\kappa_{3}>0$ and for $(i(x, r), s(x, r))=\nu^{\mathcal{C}}(x, r)$.

The existence of an ECLF on a SCC induced by the automaton $\mathcal{A}$ implies exponential stabilizability of the system (1) under language constraints. We can thus provide a solution to Problem 1 into two steps:

Step 1: Firstly, for any nontrivial SCC induced by the automaton $\mathcal{A}$, it is checked if there exists a ECLF. If there exists at least one nontrivial SCC for which 
there exists a ECLF, Problem 1 may have a solution depending on the initial automaton states.

Step 2: Secondly, we have to check if at least one initial condition can reach a SCC that admits a ECLF. The set of automaton states that can reach a given SCC is the union of all the SCC (trivial or not) that are a predecessor, in the sense of the partial relation order of the SCCs (see Definition 8), of any nontrivial SCC associated with an ECLF. In other words, if $d$ is the number of SCCs, let be $\mathcal{Q}=\left\{i \in \mathbb{N}_{d}, \mathcal{C}_{i}\right.$ admits an ECLF $\}$, then

$$
\tilde{\mathcal{S}}^{0}=\mathcal{S}^{0} \bigcap\left(\bigcup_{j \in \mathcal{Q}} \bigcup_{\substack{i \in \mathbb{N}_{d} \\ \mathcal{C}_{i} \succeq \mathcal{C}_{j}}} \mathcal{C}_{i}\right)
$$

is the set of initial automaton states that can be chosen to reach a SCC that admits an ECLF. If $\tilde{\mathcal{S}}^{0} \neq \emptyset$, then Problem 1 admits a solution.

The second step can be viewed as the reachability on a digraph, which is decomposed into SCCs. There are several dedicated algorithms in graph theory. One of the most reknown is the Dijkstra's algorithm [5]. Such an algorithm may be considered to build a finite path between any $s \in \tilde{\mathcal{S}}^{0}$ (defined by Equation (7)) and an arbitrary $r \in \bigcup_{j \in \mathcal{Q}} \mathcal{C}_{j}$. This path is the prefix of a stabilizing switching law verifying the constrained language $L(\mathcal{A})$.

Solving Problem 1 is reduced to solve the first step. That is the goal of the following section.

\section{APPROACH BASED ON LyAPUNOV-METZLER INEQUALITIES}

This section is focused on providing a solution for Step 1 with an approach considering Lyapunov-Metzler inequalities taking into account the language constrained $L(\mathcal{A})$.

\section{A. Lyapunov-Metzler inequalities to obtain an ECLF on a $S C C$}

This subsection is dedicated to obtain sufficient conditions for the stabilizability of a SCC induced by the automaton $\mathcal{A}$. These sufficient conditions are expressed as LyapunovMetzler inequalities. This result is gathered in Theorem 1 that provides also the related control policy over this SCC.

Without loss of generality for analogous reasons than in [8], we will consider the following condition concerning the system.

Assumption 2: The matrices $A_{i}\left(i \in \mathbb{N}_{q}\right)$ are nonsingular.

Theorem 1: Let be $\mathcal{C}$ a nontrivial SCC induced by the automaton $\mathcal{A}$, containing $h=|\mathcal{C}|$ automaton states, denoted $\mathcal{C}=\left\{c_{1}, \cdots, c_{h}\right\}$. If there exist a rectangular Metzler matrix, admissible with the $\operatorname{SCC} \mathcal{C}$, that is $\Pi \in \mathbb{R}^{q h \times h}$ with $\Pi^{\prime} \in$ $\mathcal{M}_{h \times q h}$ and $h$ symmetric positive definite matrices $M_{i}, i \in$ $\mathbb{N}_{h}$, such that the $h$ bilinear matrix inequalities

$$
\forall j \in \mathbb{N}_{h}, M_{j}>\sum_{\substack{(i, \ell) \in \mathbb{N}_{h} \times \Sigma \\ c_{i} \in \delta\left(c_{j}, \ell\right) \cap \mathcal{C}}} \pi_{i+(\ell-1) h, j} A_{\ell}^{\prime} M_{i} A_{\ell},
$$

are satisfied, then $\mathcal{C}$ admits an ECLF on the form

$$
V_{\min }:\left\{\begin{array}{lll}
\mathcal{C} \times \mathbb{R}^{n} & \longrightarrow & \mathbb{R}^{+}, \\
\left(c_{j}, x\right) & \longmapsto & \min _{\substack{(i, \ell) \in \mathbb{N}_{h} \times \Sigma \\
c_{i} \in \delta\left(c_{j}, \ell\right) \cap \mathcal{C}}} V\left(c_{i}, A_{\ell} x\right),
\end{array}\right.
$$

where

$$
V:\left\{\begin{array}{lll}
\mathcal{C} \times \mathbb{R}^{n} & \longrightarrow & \mathbb{R}^{+}, \\
\left(c_{i}, x\right) & \longmapsto & x^{\prime} M_{i} x .
\end{array}\right.
$$

Moreover, after an (arbitrary) prefix allowing to reach in finite time an automaton state $c_{i_{0}} \in \mathcal{C} \subseteq \mathcal{S}\left(i_{0} \in \mathbb{N}_{h}\right)$ from $s_{0} \in \mathcal{S}^{0}$, that is there exists $K \in \mathbb{N}$ such $s_{K}=c_{i_{0}}$, a min-switching strategy taking into account the language constraint is defined by

$$
\left(s_{k+1}, \sigma(k)\right)=\nu^{\mathcal{C}}\left(x_{k}, s_{k}\right) \in \arg \min _{\substack{(i, \ell) \in \mathbb{N}_{h} \times \Sigma \\ c_{i} \in \delta\left(s_{k}, \ell\right)}} x_{k}^{\prime} A_{\ell}^{\prime} M_{i} A_{\ell} x_{k}
$$

and $x_{k+1}=A_{\sigma(k)} x_{k}$.

Proof: We would like to ensure $\forall(j, x) \in \mathbb{N}_{h} \times \mathbb{R}^{n}$, $x \neq 0, \exists(i, \ell) \in \mathbb{N}_{h} \times \Sigma, c_{i} \in \delta\left(c_{j}, \ell\right)$ and $V\left(c_{i}, A_{\ell} x\right)<$ $V\left(c_{j}, x\right)$. This is equivalent to

$$
\min _{\substack{(i, \ell) \in \mathbb{N}_{h} \times \Sigma \\ c_{i} \in \delta\left(c_{j}, \ell\right)}} V\left(c_{i}, A_{\ell} x\right)=\min _{\substack{(i, \ell) \in \mathbb{N}_{h} \times \Sigma \\ c_{i} \in \delta\left(c_{j}, \ell\right)}} x^{\prime} A_{\ell}^{\prime} M_{i} A_{\ell} x<V\left(c_{j}, x\right) .
$$

Due to Lyapunov-Metzler inequalities (8) related to the SCC $\mathcal{C}$ of $\mathcal{A}$, and due to the fact that $M_{i}>0_{n}\left(i \in \mathbb{N}_{h}\right)$, we have

$$
\begin{aligned}
\min _{\substack{i, \ell) \in \mathbb{N}_{h} \times \Sigma \\
c_{i} \in \delta\left(c_{j}, \ell\right)}} x^{\prime} A_{\ell}^{\prime} M_{i} A_{\ell} x \leq \sum_{\substack{(i, \ell) \in \mathbb{N}_{h} \times \Sigma \\
c_{i} \in \delta\left(c_{j}, \ell\right) \cap \mathcal{C}}} \pi_{i+(\ell-1) h, j} x^{\prime} A_{\ell}^{\prime} M_{i} A_{\ell} x \\
\quad<x^{\prime} M_{j} x=V\left(c_{j}, x\right)
\end{aligned}
$$

For $k \in \mathbb{N}$, with $k \geq K$, this implies that by applying the control policy $\left(s_{k+1}, \sigma(k)\right)=\nu^{\mathcal{C}}\left(x_{k}, s_{k}\right)$ defined by (11), we have, for $x_{k} \neq 0$ and $x_{k+1}$ given by (1)

$$
V_{\min }\left(s_{k+1}, x_{k+1}\right)<V_{\min }\left(s_{k}, x_{k}\right) \text {. }
$$

Thanks to Assumption 2, $V_{\min }(\cdot, \cdot)$ is a positive definite function with respect to the second argument, that is the system state. That concludes the proof.

Theorem 1 calls some comments.

- The inequalities (8) are bilinear matrix inequalities due to the product of a scalar (component of the rectangular Metzler matrix) and a (Lyapunov) matrix. Nevertheless when fixing the rectangular Metzler matrix, the inequalities (8) become linear. That fact allows a numerical method to solve inequalities (8) via a research in line in function of the parameters of the rectangular Metzler matrix. It should be noticed that the number of parameters to consider decreases when the number of zeros in the layout (4) increases.

- The Lyapunov-Metzler inequalities approach leads to only sufficient conditions for stabilizability when there is no language constraint. See [7] for related discussions and counterexamples. This is the same in the framework of this paper. 
- It is noteworthy that the prefix obtained by the Dijkstra's algorithm can always be formulated with a min-switching strategy because it is always possible to design outside $\mathcal{C}$ a collection of matrices $M_{i}$ enforcing this path.

\section{$B$. Recovering the results with unconstrained languages}

This subsection aims at emphasizing that applying Theorem 1 allows to recover results from the literature dealing with stabilizing switching systems without language constraints. Consider a switched system of the form (1) with unconstrained switching law. We may propose (at least) two automata.

Automaton 1: We consider only one automaton state. $\mathcal{A}=$ $\left(\left\{e_{1}\right\}, \mathbb{N}_{q}, \delta,\left\{e_{1}\right\}\right)$, where the transition map verifies $e_{1} \in$ $\delta\left(e_{1}, i\right), \forall i \in \mathbb{N}_{q}$. This automaton is depicted on the left of Figure 2.

$\mathcal{A}$ admits only a single nontrivial SCC: $\mathcal{C}_{1}=\left\{e_{1}\right\}$. By applying Theorem 1 on $\mathcal{C}_{1}, \Pi \in \mathbb{R}^{q \times 1}$, we obtain the following Lyapunov-Metzler inequality

$$
M_{1}>\sum_{\ell \in \mathbb{N}_{q}} \pi_{\ell, 1} A_{\ell}^{\prime} M_{1} A_{\ell} .
$$

The Inequality (14) is exactly the one in [10, Lemma 1]. We recall that Inequality (14) implies the existence of standard Lyapunov-Metzler inequalities and that the convex combination $\sum_{\ell \in \mathbb{N}_{q}} \pi_{\ell, 1} A_{\ell}$ is Schur.

Automaton 2: We consider $q$ automaton states. $\mathcal{A}=$ $\left(\left\{e_{1}, \cdots, e_{q}\right\}, \mathbb{N}_{q}, \delta,\left\{e_{1}, \cdots, e_{q}\right\}\right)$, where the transition map verifies $\left\{e_{i}\right\}=\delta\left(e_{j}, i\right), \forall(i, j) \in \mathbb{N}_{q}^{2}$. In other words, the arrival of a transition is indexed by the mode $i$. This automaton is depicted on the right of Figure 2.

$\mathcal{A}$ admits only a single nontrivial SCC: $\mathcal{C}_{1}=\left\{e_{1}, \cdots, e_{q}\right\}$. By applying Theorem 1 on $\mathcal{C}_{1}, \Pi \in \mathbb{R}^{q^{2} \times q}$, we obtain the following Lyapunov-Metzler inequalities

$$
\forall j \in \mathbb{N}_{q}, M_{j}>\sum_{\ell \in \mathbb{N}_{h}} \pi_{\ell+(\ell-1) q, j} A_{\ell}^{\prime} M_{\ell} A_{\ell} .
$$

Since the rectangular Metzler matrix is admissible with the single nontrivial SCC of $\mathcal{A}$, only $q$ lines among $q^{2}$ can be not trivial (namely the index of these lines are $\{i(q+1)-q\}_{i \in \mathbb{N}_{q}}$ ). By erasing these trivial lines, we can build a square extraction of $\Pi, \tilde{\Pi} \in \mathbb{R}^{q \times q}$, which is a square Metzler matrix $\tilde{\Pi}^{\prime} \in$ $\mathcal{M}_{q \times q}$, such that

$$
\tilde{\pi}_{\ell, j}=\pi_{\ell+(\ell-1) q, j}, \forall(\ell, i) \in \mathbb{N}_{q}^{2} .
$$

By introducing $P_{i}=A_{i}^{\prime} M_{i} A_{i}, \forall i \in \mathbb{N}_{q},\left(P_{i}>0_{n}\right.$, thanks to Assumption 2 the inequality (15) reads

$$
\begin{aligned}
P_{j}=A_{j}^{\prime} M_{j} A_{j} & >\sum_{\ell \in \mathbb{N}_{q}} \tilde{\pi}_{\ell, j} A_{j}^{\prime} A_{\ell}^{\prime} M_{\ell} A_{\ell} A_{j} \\
& =A_{j}^{\prime}\left(\sum_{\ell \in \mathbb{N}_{q}} \tilde{\pi}_{\ell, j} P_{\ell}\right) A_{j}, \forall j \in \mathbb{N}_{q},
\end{aligned}
$$

which are the standard Lyapunov-Metzler inequalities [10].

\section{ILLUSTRATIONS}

Example 3: Let us consider the switched system (1) with $q=3$ modes, $x_{0}^{\prime}=\left(\begin{array}{cc}1 & -1\end{array}\right)^{\prime}$ and

$\left[\begin{array}{lll}A_{1} & A_{2} & A_{3}\end{array}\right]=\left[\begin{array}{cc|cc|cc}0.9 & 0 & 0.6 & 0 & 1.2 & 1 \\ 0 & 0.7 & 0 & 1 / 0.6 & 0 & 0.8\end{array}\right]$.
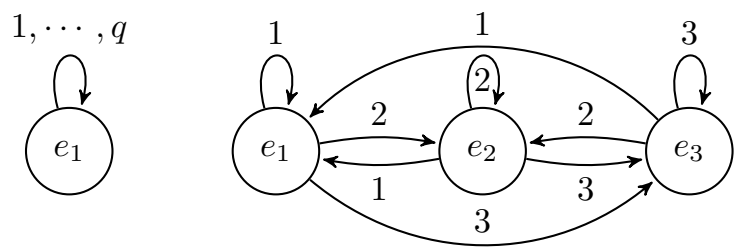

Fig. 2. Automatons 1 with $q=1$ (left) and 2 with $q=3$ (right).

The automaton defining the constrained language is depicted on Figure 3, with $\mathcal{S}^{0}=\left\{e_{1}, e_{2}, e_{4}\right\}$. It consists of $p=6$ states and admits $d=4$ SCCs which can be described as follows:

- $\mathcal{C}_{1}=\left\{e_{1}\right\}$ is a trivial SCC.

- $\mathcal{C}_{2}=\left\{e_{5}, e_{6}\right\}$ is a nontrivial SCC. Inequalities (8) admit (at least) a solution. For instance, we have

$$
\begin{gathered}
M_{5}=\left[\begin{array}{ll}
0.0407 & 0.1665 \\
0.1665 & 2.4735
\end{array}\right] ; M_{6}=\left[\begin{array}{ll}
0.0167 & 0.1668 \\
0.1668 & 6.5058
\end{array}\right] \\
\text { and } \begin{aligned}
M_{5} & >0.4 A_{1}^{\prime} M_{6} A_{1}+0.6 A_{3}^{\prime} M_{5} A_{3}, \\
M_{6} & >0.8 A_{2}^{\prime} M_{5} A_{2}+0.2 A_{3}^{\prime} M_{6} A_{3} .
\end{aligned}
\end{gathered}
$$

- $\mathcal{C}_{3}=\left\{e_{4}\right\}$ is a nontrivial SCC. $A_{2}$ being unstable, Inequality (8) associated with $\mathcal{C}_{3}$ cannot admit a solution.

- $\mathcal{C}_{4}=\left\{e_{2}, e_{3}\right\}$ is a nontrivial SCC. The only possible cycle is a periodic one and $A_{2} A_{3}$ is not stable. Inequalities (8) associated with $\mathcal{C}_{4}$ cannot admit a solution.

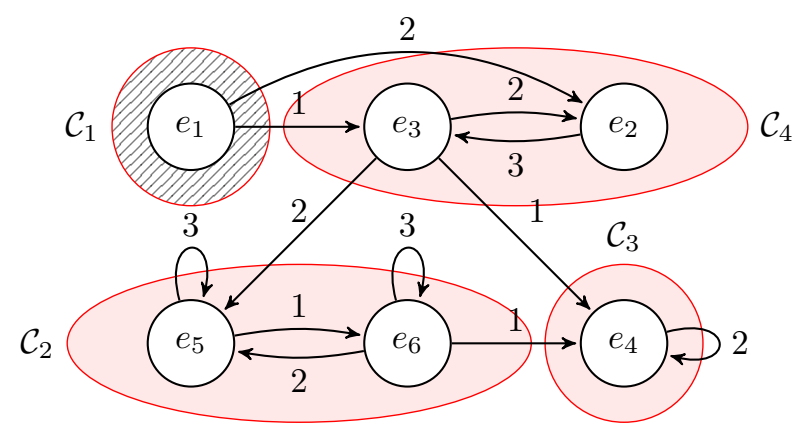

Fig. 3. Automaton related to Example 3 and its related SCCs: the trivial SCC are depicted with a pattern and the nontrivial SCC are colored in red.

Thanks to the DAG gathering the SCCs, we have $\mathcal{C}_{1} \succeq$ $\mathcal{C}_{4} \succeq \mathcal{C}_{2} \succeq \mathcal{C}_{3}$. Every vertex of $\mathcal{C}_{2}$ can be reached by every vertex of $\mathcal{C}_{2} \cup \mathcal{C}_{4} \cup \mathcal{C}_{1}$. In order to obtain a prefix of a stabilizing control policy, we have to select as initial vertex an element of $\mathcal{S}^{0} \bigcap\left(\mathcal{C}_{2} \cup \mathcal{C}_{4} \cup \mathcal{C}_{1}\right)=\left\{e_{1}, e_{2}\right\}$, which is not empty. The system is thus stabilizable and there is a solution to Problem 1.

For the prefix of the stabilizing switching law, we choose $e_{1} \in \mathcal{S}^{0} \bigcap\left(\mathcal{C}_{2} \cup \mathcal{C}_{4} \cup \mathcal{C}_{1}\right)$ as initial vertex of the $e_{6} \in \mathcal{C}_{2}$ to be reached by the prefix. The Dijkstra path is then given by:

$$
e_{1} \stackrel{\sigma(0)=1}{\longrightarrow} e_{3} \stackrel{\sigma(1)=2}{\longrightarrow} e_{5} \stackrel{\sigma(2)=1}{\longrightarrow} e_{6},
$$

with $K=3$. For $k \geq K$, we can thus apply the minswitching control policy defined by (11) resulting in the 
trajectory in the system state space and in the automaton state space depicted in Figure 4. Moreover the switching law induced by this control policy is depicted on Figure 5, in addition of the min-switching Lyapunov function $V_{\min }\left(s_{k}, x_{k}\right)$, for $k \geq K+1$. We can observe that the Lyapunov function decreased as expected.
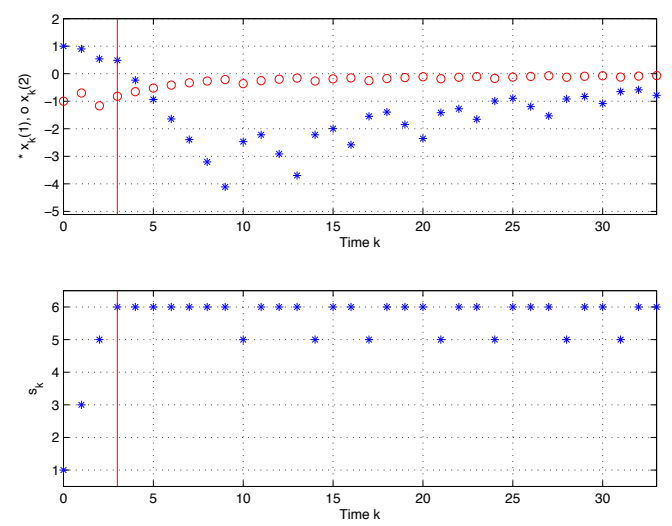

Fig. 4. Trajectories $k \mapsto x_{k}$ and $k \mapsto s_{k}$ related to Example 3. The vertical red line split in time the Dijkstra path and the application of min-switching strategy.
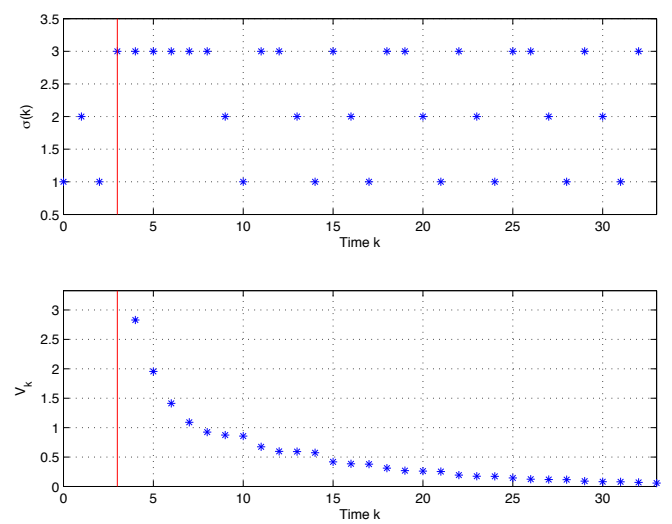

Fig. 5. Functions $k \mapsto \sigma(k)$, for $k \in \mathbb{N}$ and $k \mapsto V_{k}$, for $k \geq K+1$ related to Example 3. The vertical red line split in time the Dijkstra path and the application of min-switching strategy.

\section{CONCLUSION}

In this paper, the stabilizability of an autonomous discretetime switched system with language constraints concerning the switching law has been investigated. Based on a decomposition into strongly connected components of the automaton generating the constrained language and based on their properties, a methodology has been applied to consider only the stabilizability on strongly connected components. Afterwards, a Lyapunov-Metzler inequalities approach has been applied to check the existence of an exponentially stabilizing control Lyapunov function over each strongly connected component. The stabilizability is obtained thanks to a prefix given by the Dijkstra algorithm plus a minswitching strategy induced by the exponentially stabilizing control Lyapunov function over at least one strongly connected component. Several numerical and generic examples have been presented to illustrate the contribution.

\section{REFERENCES}

[1] C. Baier and J.-P. Katoen. Principles of Model Checking. The MIT Press, 2008.

[2] J. Daafouz, P. Riedinger, and C. Iung. Stability analysis and control synthesis for switched systems : A switched Lyapunov function approach. IEEE Transactions on Automatic Control, 47:1883-1887, 2002.

[3] J. Daafouz, S. Tarbouriech, and M. Sigalotti, editors. Hybrid Systems with Constraints. Wiley-ISTE, July 2013.

[4] R. Diestel. Graph Theory. Springer-Verlag, New York, electronic edition, 2000.

[5] E. Dijkstra. A Discipline of Programming. NJ: Prentice Hall, 1976.

[6] C. Duan and F. Wu. Dwell-time min-switching for discrete-time switched linear systems. In Proceedings of the European Control Conference, pages 2540-2545, Strasbourg, France, 2014.

[7] M. Fiacchini, A. Girard, and M. Jungers. On the stabilizability of discrete-time switched linear systems: Novel conditions and comparisons. IEEE Transactions on Automatic Control, 61(5):1181-1193, 2016.

[8] M. Fiacchini and M. Jungers. Necessary and sufficient condition for stabilizability of discrete-time linear switched systems: a set-theory approach. Automatica, regular paper, 50(1):75-83, January 2014.

[9] M. Fiacchini, M. Jungers, and A. Girard. Exponential stabilization of language constrained discrete-time switched linear systems: a geometrical approach. In Proceedings of the European Control Conference, 2016.

[10] J. C. Geromel and P. Colaneri. Stability and stabilization of discretetime switched systems. International Journal of Control, 79(7):719728, July 2006.

[11] L. Gurvits. Stability of discrete linear inclusion. Linear Algebra and its Applications, 231:47-85, 1995.

[12] J. Hespanha and A. Morse. Stability of switched systems with average dwell-time. In Proceedings of 38th IEEE Conference on Decision and Control, pages 2655-2660, 1999.

[13] R. M. Jungers and P. Mason. On feedback stabilization of linear switched systems via switching signal control. 2016. http://arxiv.org/abs/1601.08141.

[14] Ö. Karabacak. Dwell time and average dwell time methods based on the cycle ratio of the switching graph. Systems \& Control Letters, 62:1032-1037, 2013.

[15] J. W. Lee and G. E. Dullerud. Uniformly stabilizing sets of switching sequences for switched linear systems. IEEE Transactions on Automatic Control, 52:868-874, 2007.

[16] D. Liberzon. Switching in Systems and Control. Systems and Control: Foundations and Applications. Birkhäuser, 2003.

[17] H. Lin and P. J. Antsaklis. Stability and stabilizability of switched linear systems: A survey of recent results. IEEE Transactions on Automatic Control, 54(2):308-322, 2009.

[18] J. L. Mancilla-Aguilar, R. Garcia, E. Sontag, and Y. Wang. Uniform stability properties of switched systems with switchings governed by digraphs. Nonlinear Analysis, 63:472-490, 2005.

[19] M. Philippe, G. Millérioux, and R. M. Jungers. Deciding the boundedness and dead-beat stability of constrained switching systems. Nonlinear Analysis: Hybrid Systems, to appear, 2016. http://arxiv.org/abs/1512.04887.

[20] K. Ruohonen. Graph Theory. Tampere University of Technology, 2008.

[21] E. De Santis and M. D. Di Benedetto. Theory and computation of discrete state space decompositions for hybrid systems. European Journal of Control, 19(1):1-10, 2013.

[22] M. Sharir. A strong connectivity algorithm and its applications to data flow analysis. Computers and Mathematics with Applications, 7(1):67-72, 1981

[23] E. Skafidas, R. J. Evans, A. V. Savkin, and I. R. Petersen. Stability results for switched controller systems. Automatica, 35(4):553-564, 1999.

[24] R. E. Tarjan. Depth-first search and linear graph algorithms. SIAM Journal on Computing, 1(2):146-160, 1972.

[25] Y. Wang, N. Roohi, G. E. Dullerud, and M. Viswanathan. Stability analysis of switched linear systems defined by regular languages. In Proceedings of the 53rd Conference on Decision and Control, pages 5445-5450, Los Angeles, CA, USA, 2014.

[26] W. Zhang, A. Abate, J. Hu, and M. P. Vitus. Exponential stabilization of discrete-time switched linear systems. Automatica, 45(11):25262536, 2009. 